\title{
DATA-DRIVEN DESIGN-BY-ANALOGY: STATE OF THE ART AND FUTURE DiRECTIONS
}

\author{
Shuo Jiang* \\ Shanghai Jiao Tong University \\ 800 Dongchuan Road, Shanghai, China, 200240 \\ jsmech@sjtu.edu.cn \\ Kristin L. Wood \\ University of Colorado Denver \\ 1201 Larimer St, Denver, CO, USA, 80204 \\ kristin.wood@ucdenver.edu
}

\author{
Jie Hu \\ Shanghai Jiao Tong University \\ 800 Dongchuan Road, Shanghai, China, 200240 \\ hujie@sjtu.edu.cn \\ Jianxi Luo \\ Singapore University of Technology and Design \\ 8 Somapah Road, Singapore, 487372 \\ luo@sutd.edu.sg
}

June 1, 2021

\begin{abstract}
Design-by-Analogy (DbA) is a design methodology wherein new solutions, opportunities or designs are generated in a target domain based on inspiration drawn from a source domain; it can benefit designers in mitigating design fixation and improving design ideation outcomes. Recently, the increasingly available design databases and rapidly advancing data science and artificial intelligence technologies have presented new opportunities for developing data-driven methods and tools for DbA support. In this study, we survey existing data-driven DbA studies and categorize individual studies according to the data, methods, and applications in four categories, namely, analogy encoding, retrieval, mapping, and evaluation. Based on both nuanced organic review and structured analysis, this paper elucidates the state of the art of data-driven DbA research to date and benchmarks it with the frontier of data science and AI research to identify promising research opportunities and directions for the field. Finally, we propose a future conceptual data-driven DbA system that integrates all propositions.
\end{abstract}

Keywords Data-driven Design · Analogy $\cdot$ Design-by-Analogy $\cdot$ Artificial Intelligence $\cdot$ Data Science

\section{Introduction}

Design-by-Analogy (DbA) is a design methodology, wherein new solutions are generated in a target domain based on inspiration drawn from a source domain through cross-domain analogical reasoning [1, 2, 3]. DbA is an active research area in engineering design and various methods and tools have been proposed to support the implement of its process [4, 5, 6, 7, 8]. Studies have shown that $\mathrm{DbA}$ can help designers mitigate design fixation [9] and improve design ideation outcomes [10].

Fig.1 presents an example of DbA applications [11]. This case aims to solve an engineering design problem: How might we rectify the loud sonic boom generated when trains travel at high speeds through tunnels in atmospheric conditions [11, 12]? For potential design solutions to this problem, engineers explored structures in other design fields than trains or in the nature that effectively "break" the sonic-boom effect. When looking into the nature, engineers discovered that kingfisher birds could slice through the air and dive into the water at extremely high speeds to catch prey while barely making a splash. By analogy, engineers re-designed the train's front-end nose to mimic the geometry of the kingfisher's beak. This analogical design reduced noise and eliminated tunnel booms. It also allowed the train to travel $10 \%$ faster

${ }^{*}$ Contact email: jsmech@sjtu.edu.cn 


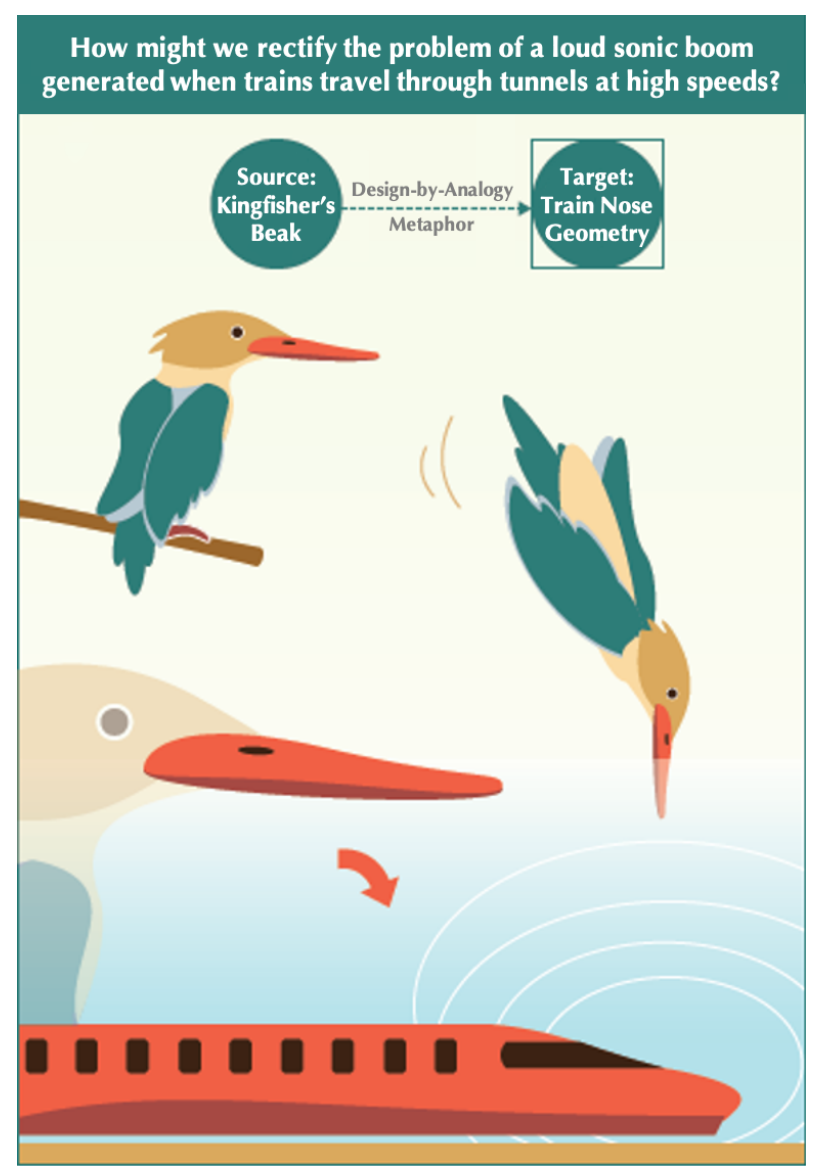

Figure 1: Exemplar of DbA: high speed train nose design inspired by kingfisher's beak [11]

consuming $15 \%$ less energy ${ }^{2}$ The example shows a practical case where design-by-analogy leads to creative problem solving and dramatic performance improvement.

In recent years, the increasingly available design databases and rapidly advancing data science and artificial intelligence (AI) technologies have enabled new data-driven methods and tools that support DbA. For example, deep learning, knowledge graph, natural language understanding, and computer vision may support the analogy representation, retrieval, mapping, and evaluation processes [13, 14, 15, 16, 17, 18, 19]. To the best of our knowledge, there is no systematic review and structured analysis of the literature on data-driven DbA to date. Therefore, to reveal promising research opportunities and future directions, we reviewed the state-of-the-art data-driven methods and tools for $\mathrm{DbA}$ and benchmarked them with the latest data science and AI technologies.

This paper proceeds as follows. Section 2 provides the theoretical background of DbA, including the analogical thinking models in cognitive psychology and experimental understanding of the effect of DbA on creativity in the engineering design field. Section 3 describes the research methodology. Section 4 presents a nuanced organic review of the data-driven DbA research literature individually. Section 5 provides a structured and integrated analysis of all the literature together, from three perspectives: data, methods, and applications, to reveal the general state-of-the-art of the field. Section 6 maps the course of feasible directions for future research from three aspects and their interactions. Finally, section 7 concludes the paper.

\section{Background}

Cognitive psychology reveals that people often tend to solve given problems by mapping solutions from known questions [20, 21, 22, 23, 24, 25], i.e., analogical reasoning. Markman [21, 26] describes analogy as a powerful cognitive mechanism that allows two objects to be seen as similar based on the connected systems of relations

\footnotetext{
${ }^{2}$ https://asknature.org/innovation/high-speed-train-inspired-by-the-kingfisher/
} 
between them. Making analogical inferences can improve critical thinking and logical reasoning [22]. Gentner et al. [27] suggested that making analogy is a key ability that distinguishes humans from other species and introduced a three-phase model of the analogical reasoning process including retrieval, mapping, and evaluation. Although other researchers proposed slightly different subdivisions, they broadly agreed on these phases [22, 23, 24]. Human analogical processes generally begin with storing and encoding the source knowledge (analogy candidates) of memory, followed by recognizing and searching the source analogy based on the given target problem. The source analogy then serves as the base model through which structured knowledge can be understood, applied, or projected on to a less familiar or abstract domain [28, 29]. In the retrieval and mapping processes, three types of similarities are usually exploited, namely, literal, attributional, and relational similarities [30]. Prior studies have revealed that a good analogy often involves an alignment of relational structure [31]. After mapping, the final step, i.e., evaluation is performed, which entails judging the analogy along with any generated inferences. In addition to the above-mentioned steps, other subphases, such as abstraction and parallel subprocess interaction, have also been described as essential cognitive processes in analogical reasoning [32].

Since the previous century, several computation-based models of analogy-making have been proposed [33, 34, 35, 36]. French [37] classified these models into three broad groups based on their underlying architecture. (1) Symbolic models are largely part of the symbolic paradigm in the AI field, in which symbols, logic, search, planning, and means-ends analysis, play an essential role. One of the most famous symbolic models is ANALOGY [33], proposed by Evans, which was designed to conduct proportional analogies of the form "A:B::C:?" obtained from the test questions of geometric figures. (2) Connectionist models adopt the framework of complex networks, including nodes, edges, and their weights. For example, the LISA analogy engine [34] relies on partially distributed representations of concepts, selective activation, and dynamic binding to associate the relevant structures to support both analogy-retrieval and mapping. (3) Hybrid models share the features of both connectionist and symbolic models, and they typically comprise neurons that enable symbolic interpretation or interactions. For instance, the AMBR model [35] consists of many micro-agents, each of which represents a small piece of knowledge that enables analogy-making.

Using analogical reasoning to generate new design solutions or concepts is referred as Design-by-Analogy in the field of engineering design. Gill et al. [38] and Tsoka et al. [39] investigated the dimensions of product similarity that designers tend to use to identify the source product when using $\mathrm{DbA}$ in engineering design. These empirical studies indicate designers utilize at least six different dimensions to draw analogy between target and source products: working principle, structure, human interaction, function, energy flows, and material flows. Among them, working principle, structure, and human interaction are more dominant in driving analogy than the other dimensions. In addition, engineering design researchers have examined how the analogical distance [10, 40, 41, 42, 43, 44, 45], representation of modality [42, 46, 47], commonness [42], amount or quantity [48], time of involvement of analogy [49], and type of similarity [50, 51] influence the solutions or concepts generated using the DbA. Among these factors, the influence of the analogical distance between the source and target domains has received the most attention.

Recent researchers have advanced the understanding of the multifaceted effects of the analogical distance on ideation behaviors and outcomes. The conceptual leap hypothesis states that distant analogies give rise to novelty and breakthroughs with lower efficacy, owing to surface dissimilarities [52]. This statement was corroborated by several empirical results from human-based studies. For example, according to Chan et al. [42], far-field stimuli yields more novel but fewer ideas than the near-field stimuli. Srinivasan et al. [40] stated that with the increase in the analogical distance, the novelty of the newly generated ideas increases, while both the quantity and quality decrease. Within the different experimental and empirical contexts, Fu et al. [10] observed that a moderate analogical distance might provide trade-offs and yield optimal design ideation performance.

Despite its usefulness, the efficacy of DbA is naturally limited by the designer's prior knowledge and memory for potential retrieval and mapping to a design problem. This challenge is greater for inexperienced or specialized designers with a limited scope of knowledge. Meanwhile, not every designer is proficient in analogical thinking. In fact, these conditions suggest the fundamental values of using external knowledge database as the source and using intelligent algorithms to carry out analogical reasoning. Then, the nuanced experimental findings on the analogical distance and other aspects, as reviewed in this section, may guide the retrieval of design analogies from a large-scale design database and the analogical reasoning process in data-driven workflows.

\section{Research Methodology}

This paper aims to elucidate the state-of-the-art of data-driven DbA research to date, especially when it comes to the application of AI and data science technologies. An advanced search in the Web-of-Science database was conducted to 


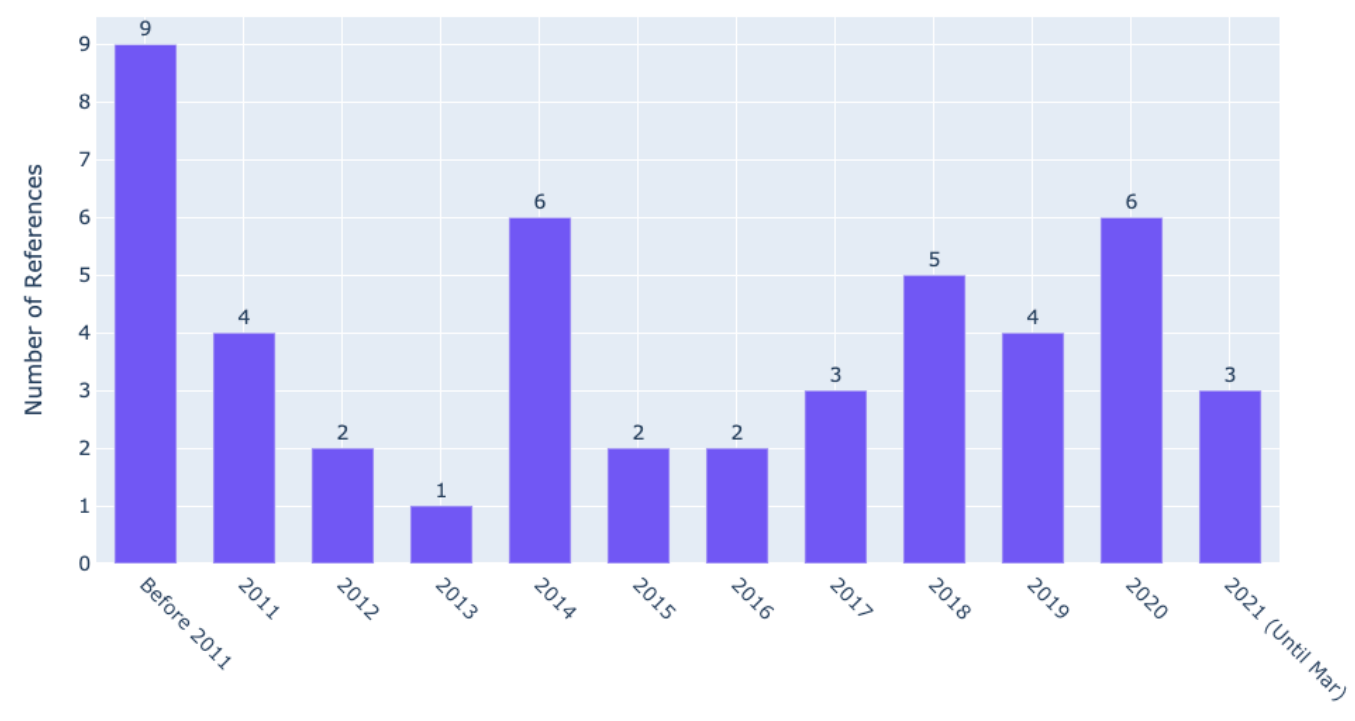

Figure 2: Number of data-driven DbA publications per year

generate an initial list of data-driven DbA publication 3 With this search query "TS $=(($ Design-by-Analogy) AND data) AND PY=1950-2021',4, we identified 23 relevant papers.

We manually examined the content of these publications and filtered out 4 articles that did not fall within the ambit of the engineering or innovation field and 6 articles that do not present data-driven methods or tools for DbA. Among the remaining ones, 5 of them are short version conference papers of the full version journal papers and are also excluded. Meanwhile, we found 39 additional relevant studies on data-driven DbA methods or tools by a snowballing search through the backward and forward citations of all the papers in the initial set (including those excluded ones in the previous step). For example, we were able to identify some recent efforts on data-driven DbA support tools, such as Idea-Inspire 4.0 [53], TechNet [54], and B-link [55], from the forward citations of a paper titled "Function-based design-by-analogy: a functional vector approach to analogical search" [5]. From the backward citation of another paper titled "Identifying candidates for design-by-analogy" [30], we could identify the first generation of DbA systems, such as KRITIK [56] and IDEAL [57]. Such newly identified studies directly refer to design-by-analogy or its synonym in the body text or discuss its usage to support one or more DbA subprocesses.

After these steps, the final literature set includes 47 papers $5^{5}$ As observed in Fig. 2 the number of publications exhibited an upward trend in recent years, indicating a growing interest in this field. Fig. 3 shows the proportional breakdown of publications by types, including journal articles, conference papers, book chapters, and patents. In Fig. 3, we marked out four journals and two conferences that include more than one papers in the final set:

Journals - (1) Journal of Mechanical Design, (2) Artificial Intelligence for Engineering Design, Analysis and Manufacturing, (3) Research in Engineering Design, and (4) Design Science.

Conferences - (1) International Design Engineering Technical Conferences \& Computers and Information in Engineering Conference (IDETC/CIE) held by ASME and (2) Conference on Human Factors in Computing Systems (CHI) held by ACM.

\section{Data-driven DbA methods and tools: an organic review}

The first generation of DbA systems, most of which were knowledge-based expert systems, were developed before the 2000s. Huhns and Acosta [58] developed the ARGO, which represents problem-solving plans using rule-based graphs that enable analogical retrieval for solving new problems. Goel et al. [56] proposed KRITIK, which represents design cases through function-behavior-structure (FBS) models [59]. KRITIK retrieves specific designs based on the

\footnotetext{
${ }^{3}$ Literature retrieval date: Feb 18, 2021.

4 "TS" stands for "topic" and "PY" stands for "published year" in the Web-of-Science search query.

${ }^{5}$ The final literature set includes these references: [4-7,13,15-19,30,41,50,53-60,62-72,77-89,91,92].
} 


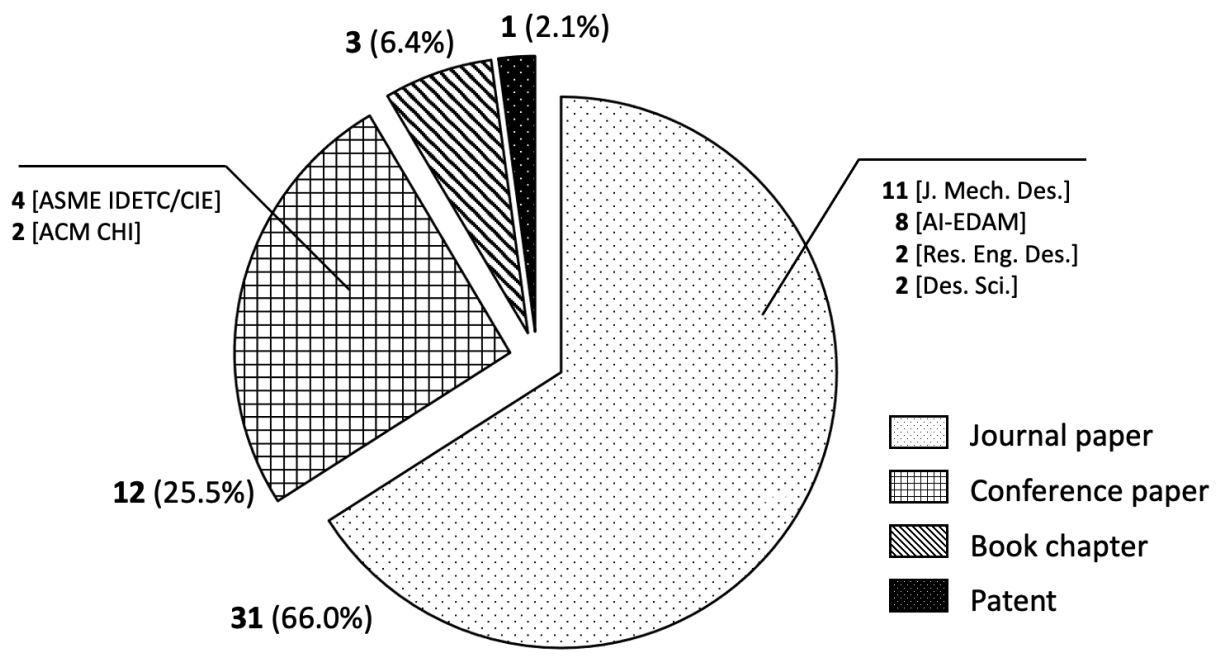

Figure 3: Distribution of data-driven DbA publications by literature types

functional information of query design, suggests modifications, and allows the verification of the newly generated designs. The same research group developed the IDEAL [57], which is also based on the FBS models. The IDEAL system allows the extraction of generic teleological mechanisms and performs analogical mapping by comparing two designs with different patterns.

With the rise of data science in the past two decades, various data-driven methods and tools have been developed to support $\mathrm{DbA}$. Reich and Shai [60] proposed the infused design method that represents design problems at the meta-level, to facilitate the discovery and use of knowledge across different technological domains. Based on the infused design theory, the interdisciplinary engineering knowledge genome (IEKG) was developed to support the search for general knowledge and method structures in the interdisciplinary domains [60]. Verhaegen et al. [30] distilled product features using the word co-occurrence analysis and principal component analysis (PCA), to automatically and systematically retrieve candidate products for DbA. Linsey et al. [4] proposed the WordTree method to linguistically re-represent design problems based on a predefined large-scale semantic network (WordNet) that can help designers seek potential analogies for idea generation. Murphy et al. [5] proposed a functional vector method to represent design documents into vectors based on the word frequency of functional verbs from the Functional Basis model [61]. A further investigation has revealed that functionally novel concepts can be generated using the functional analogy search method [50].

Sanaei et al. [62] developed an analogical retrieval method, whereby text mining and a recurrent neural network (RNN)-based ensemble model are used to extract relational structure from the text. Gilon et al. [63] proposed an analogical search engine to search distant but relevant design stimuli for specific needs using the information extraction strategy and bidirectional RNN deep learning model. Chan et al. [64] developed a mixed initiative system named SOLVENT to identify analogies among scientific papers. The system annotates four aspects of research papers (background, purpose, mechanism, and findings), and utilizes the Doc2Vec pre-trained word embeddings to represent and map different contents. Han et al. [18, 17] developed a series of computer-based tools to support DbA, including the retriever and combinator. The retriever takes ConceptNet as the knowledge base to construct the base ontology for a given design problem and search for less familiar target ontology for design ideation [18]. The combinator generates combinational prompts in both text and image forms by combining indirectly related ideas based on a customized design knowledge database constructed using ConceptNet through web crawling and text mining [17].

Among the various types of engineering design-related data sources, the patent database has attracted significant scholarly attention because of its large size, diversity, and rich design-related information in its documents. Altshuller [65] extracted various heuristic design rules, or principles, by analyzing abstract patterns in the global patent database and developed a problem-solving framework named TRIZ to guide engineers to overcome impasses in analogical reasoning. Subsequently, various data-driven tools have been proposed to support the use of TRIZ. Cascini and Russo [66] developed a system named PAT-ANALYZER to automatically construct design contradictions in TRIZ by mining patent textual information. Vincent et al. [67] proposed Bio-TRIZ, which expands on the biological information and principals in the TRIZ system. Beyond TRIZ-related tools, McCaffrey and Spector [68] developed Analogy Finder, a 
DbA support system to identify adaptable analogies in the patent database by rephrasing the description of the design problem as verbs and their synonyms. Luo et al. [15] developed InnoGPS, a computer-aided ideation tool based on the total technology space constructed in the entire United States Patent and Trademark Office (USPTO) database. The InnoGPS system can guide designers to search and retrieve knowledge and concepts for drawing analogies across domains based on knowledge distance. Similarly, Song et al. [41] utilized the technological network to locate identified patent analogies in home-, near-, and far-field stimuli based on the clustering partition results.

Recently engineering design researchers have used the latest natural language processing (NLP) and text mining techniques to construct semantic networks from engineering design-related databases, such as B-link [55] based on approximately one million engineering papers, and TechNet [54] based on six million patent documents. Both B-Link and TechNet have been used for analogical retrieval [55, 69], concept generation [70, 71], and evaluation [19]. In addition, several topic modeling algorithms have been used to structure and visualize textual design database for exploration and inspiration. Fu et al. [72] utilized the combination of a Bayesian model and latent semantic analysis to map patent documents in a network structure; this method provides a basis for automated retrieval of cross-domain inspirational analogy. The analogical distance between the initial design problem and potential stimuli is defined as the path length in a Bayesian network. Song et al. [6] applied the hierarchical nonnegative matrix factorization (NMF), a topic modeling algorithm, to structurally represent patent database with three facets: behavior, material, and component. Based on this clustered structure of patent data, they developed VISION [7], a visual interaction tool for seeking analogical inspiration and data exploration.

Bio-inspired design (BID) [73, 74, 75, 76] is a form of DbA whereby inspiration is drawn from natural biological phenomenon for engineering design. In recent years, many efforts have been made to develop data-driven methods, tools, and databases for BID. Cheong et al. [77] and Nagel et al. [78] developed the engineering-to-biology thesaurus by mining meaningful keywords from biological text aligned with the engineering functional terms in the functional basis. The thesaurus serves as the basis for engineers to find biological analogies and identify the functional reasoning linking two domains when solving design problems. Goel et al. [79] developed the Design by Analogy to Nature Engine (DANE), a design case library containing approximately 40 FBS models of natural and artificial systems. DANE also provides a framework for users to retrieve previous cases based on queries in multiple forms. Helms and Goel [80] proposed the four-box method to manually represent design problems in four facets (functions, environments, specifications, and performance) based on FBS models and evaluate analogies using the heuristic T-chart in the BID. Cheong and Shu [81] proposed the syntactic rule-based computational method to identify biological analogies that involve causal relations. Glier et al. [82] explored the use of automated text classifiers for BID, including naive Bayes classifiers, k-nearest neighbors, and support vector machine, to determine whether a textual stimuli is helpful for solving the given design problem. The Biomimicry 3.8 Institute [83] developed a web-based tool, AskNature, which employs a dedicated taxonomy to manage the more than 1,600 biological cases in its repository, to provide biological analogies for engineering designers.

Verhaegen et al. [84] developed product aspects in DbA (PAnDA), a tool to identify candidate products for DbA by extracting the product aspects from texts through rule-based text-mining techniques. They also developed SEABIRD [85] based on the same strategy to map the technical systems in patent documents and biological systems in academic papers. SEABIRD enables the scalable search for biological stimuli for designers. Lucero et al. [86, 87] integrated the functional basis model, WordNet, and the AskNature repository to develop the D-APPS tool and DRACULA software, which enable analogy matching between biological and engineering concepts and analogy searching for BID. Siddharth and Chakrabarti [53] developed the Idea-Inspire 4.0 tool, which represents both biological and engineering concepts based on the domain-agnostic SAPPhIRE model ontology [88]. Idea-Inspire 4.0 supports the analogical retrieval and evaluation based on a manually created database of 60 biological concepts and 83 engineered concepts. More recently, Kruiper et al. [16, 89] built a dataset named focused open biology information extraction (FOBIE) and used it to train an NLP model to extract semi-open trade-off relations and arguments from scientific biological documents, providing a high-level filter for engineers. Bhasin et al. [90] proposed a reduced function-means tree to distill both biological adaptations and their associated product architecture from existing bioinspired design abstraction database and product architecture representation database.

Although most of the aforementioned methods and tools entailed processing textual information, visual or multimodal analogy for design inspiration has been explored in recent research. Kwon et al. [91] developed a framework to exploit visual similarity based on the web-based search engine to find visual analogies for further idea generation. Jiang et al. [13] proposed a convolutional neural network (CNN)-based model to automatically derive the vector space and design the feature vectors to represent patent images. The derived feature vectors that embed both visual information and technology-related knowledge can potentially facilitate the retrieval of visual stimuli based on the vector distances. Zhang et al. [92] proposed an unsupervised deep learning model, Sketch-pix2seq, which is trained using reconstruction and clustering losses to allow the extraction of the underlying shape features of sketches in the Quickdraw database. 


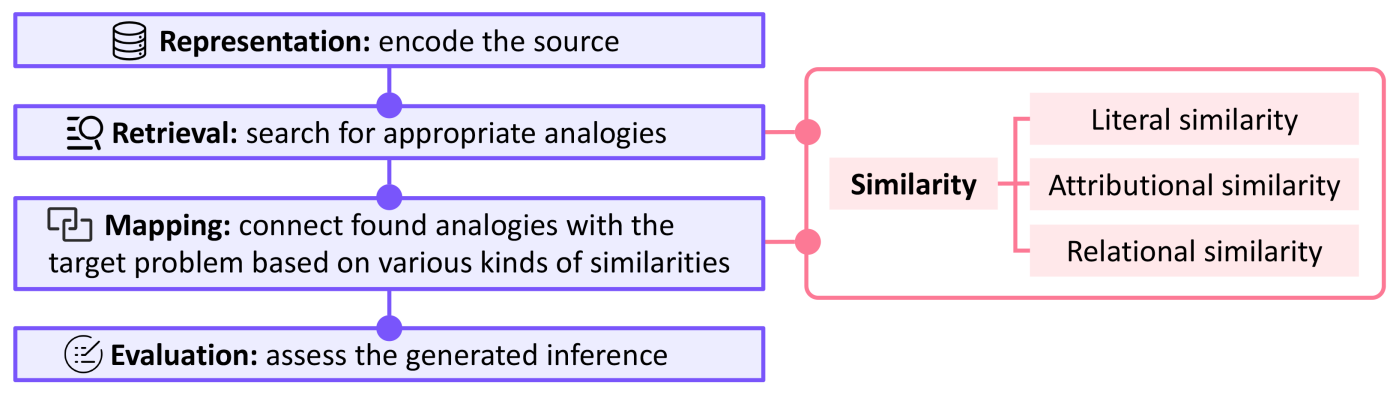

Figure 4: The four-phase model of making analogy in cognitive science

The constructed latent space for sketches provides a new way to define visual similarities and search the analogical sketches for DbA.

\section{Structure and state of the field: a synthesis}

\subsection{Overview}

In section 4, we have presented a nuanced organic review of each data-driven DbA method or tool identified via our literature search. To elucidate the general state-of-the-art of the field, this section aims to provide an integrated and structured analysis of all works in the literature together from the viewpoint of the following three aspects:

1. The databases, datasets, or repositories used for developing relevant methods or tools;

2. The application goals, which usually belong to one or more subphases of the DbA process, based on a four-phase analogy model in the cognitive science field (depicted in Fig. 4).

3. The AI or data science algorithms used in the data-driven DbA studies.

Fig. 5 presents a visual summary of the datasets, methods, applications of existing DbA studies, as well as the coupling of the three dimensions. A single DbA method or tool may contain more than one DbA subphase. We separately counted each item in Fig. 5. ensuring that each used algorithm or method was matched to the corresponding applications. The different line colors denote different phases in the DbA process. We further developed a web-based interactive data visualization ${ }^{6}$ for public users to probe such coupling relationships among the three perspectives in individual prior studies.

\subsection{Data}

Table 1 summarizes the datasets used in DbA, including databases specifically developed for DbA research. The domain-specific knowledge databases, including DANE and Idea-Inspire, were manually created based on humancoding structures. The representation of design and relevant concepts by using predefined models is a time-consuming and labor-intensive task and requires domain expertise. Consequently, these repositories are usually small scale and lack scalability. For example, in the DANE repository, building a complete FBS model of a biological system requires 40-100 hours [79]; thus, the repository contains only 40 cases and 22 complete FBS models. These models lack a uniform description and generalizability to enable processing large-scale databases, which are pre-conditions for adopting modern AI algorithms. While scholars have proposed building large and scalable digital libraries of analogical design case studies for broader usages [93], how to represent, aggregate and organize existing scattered and unstructured design data efficiently with a consistent structure remains an open question.

Compared with human-curated databases, patent and scientific paper databases are naturally structured and continually populated over time and cover a wide range of domains [54, 55]. They are public big-data sources that enable the development of scalable data-driven DbA methods and tools. Specifically, patent documents contain rich engineering design information of the structures, functions, mechanisms, and principles that might be useful for the design inspiration process [5, 13, 15, 40, 72, 94]. 


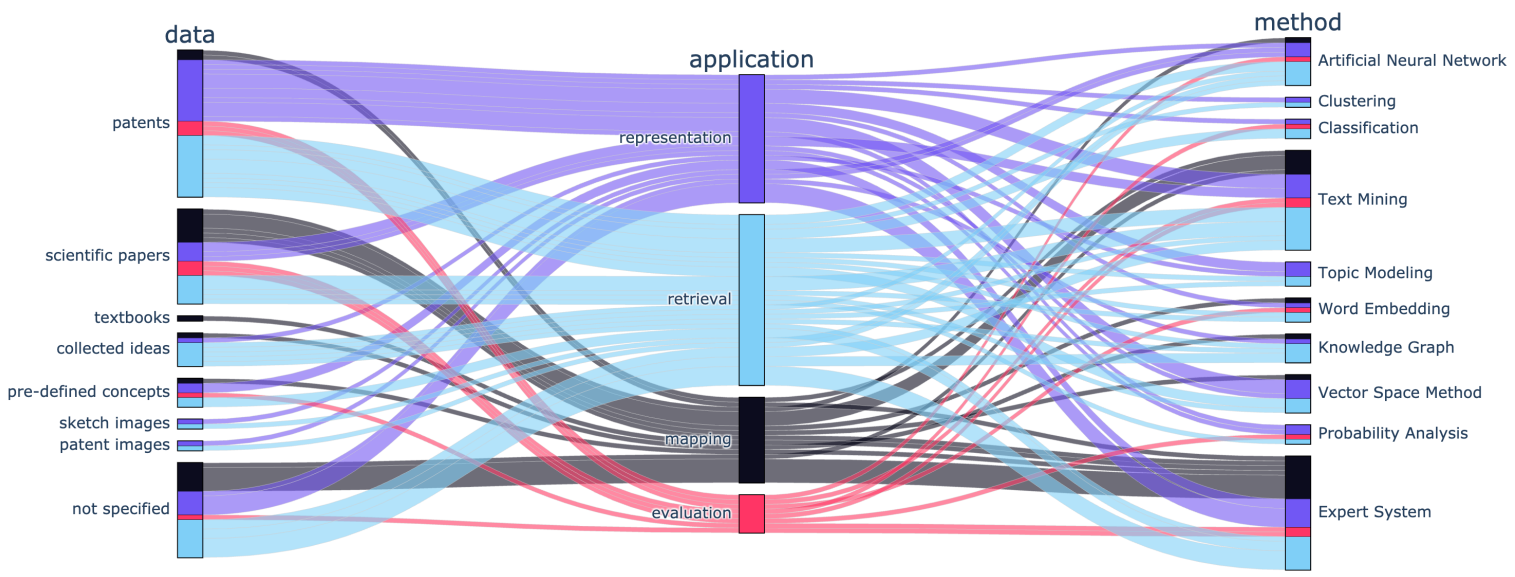

Figure 5: Overview of the state-of-the-art for data-driven DbA studies

Table 1: Existing databases that have been adopted or developed for $\mathrm{DbA}$

\begin{tabular}{|c|c|c|}
\hline Category & Data & Size and refs \\
\hline \multirow{3}{*}{ Patents } & Texts from the whole USPTO database & More than 6 million patent documents [15, 54] \\
\hline & $\begin{array}{l}\text { Texts from the subset of the USPTO } \\
\text { database }\end{array}$ & $\begin{array}{l}155,000[30,84,85], 6,500[5], 10,400[7], 200 \\
{[41], 100[72] \text { patent texts }}\end{array}$ \\
\hline & $\begin{array}{l}\text { Images from the subset of the USPTO } \\
\text { database }\end{array}$ & $\begin{array}{l}\text { Images from the subset of the USPTO database } \\
\text { [13] }\end{array}$ \\
\hline \multirow{4}{*}{$\begin{array}{l}\text { Scientific } \\
\text { paper/books }\end{array}$} & Papers from computer science conferences & Papers from computer science conferences \\
\hline & Papers from biological journals & $500[82], 8,000[85], 10,000[16]$ papers \\
\hline & Papers from engineering journals & More than 1 million papers $[55]$ \\
\hline & A biological textbook & A biological textbook [77] \\
\hline \multirow{2}{*}{$\begin{array}{l}\text { Collected } \\
\text { ideas }\end{array}$} & Concepts collected from Quirky platform & The corpus cover over 8,500 products [63] \\
\hline & Data crawled from the Internet & Unknown [17, 91$]$ \\
\hline Sketches & Quickdraw & 75,000 sketches [92] \\
\hline \multirow{4}{*}{$\begin{array}{l}\text { Biomimicry } \\
\text { databases }\end{array}$} & AskNature & Over 1,700 biological concepts [83] \\
\hline & DANE & 40 FBS-based biological systems [79] \\
\hline & Idea-Inspire & $\begin{array}{l}\text { Over } 100 \text { biological and engineering SAPPhIRE- } \\
\text { based concepts [53] }\end{array}$ \\
\hline & FOBIE & $\begin{array}{l}\text { Over } 1,500 \text { annotated sentences with } 4,700 \text { rela- } \\
\text { tions [16] }\end{array}$ \\
\hline \multirow{3}{*}{$\begin{array}{l}\text { Semantic } \\
\text { Network- } \\
\text { based } \\
\text { databases }\end{array}$} & WordNet & 155,000 entities with 648,000 relations [95] \\
\hline & ConceptNet & 517,000 entities with $1.3 \times 10^{11}$ relations $[96]$ \\
\hline & TechNet & $\begin{array}{l}\text { Over } 4 \text { million technical terms with their relation- } \\
\text { ships [54] }\end{array}$ \\
\hline
\end{tabular}


Recently, extensive research has established that crowdsourced design solution data collected from online ideation and task-orientated platforms (e.g., InnoCentive, Quirky, OpenIDEO, and Amazon Mechanical Turk) are useful for DbA research [97, 98, 99]. The crowdsourced solutions make it possible to mine the real-world ideation outputs of complex cognitive tasks generated by thousands of people across the world. In addition, some researchers have observed that crowdfunding platforms (e.g., Kickstarter and Indiegogo) also accumulate product design projects and contain rich design information about product designs, and are therefore potentially useful data sources for analogical design stimuli [100].

Majority of the existing DbA research only focused on textual data and ignored data in other modalities such as visual information. Recently, some scholars have explored the mining of the large-scale 2D design image datasets (such as patent images and sketches) using various computer vision techniques. Other 2D image datasets and 3D model datasets, such as Shape-Net [101], the Thingiverse repository 7 , the IKEA design dataset [102], and mechanical CAD model dataset [103], can be explored and utilized to develop tools for supporting DbA with visual analogies or multimodal analogies.

Furthermore, there is a paucity of gold-standard benchmarking datasets developed for specific DbA tasks. Consequently, the comparison and evaluation of different methods and tools in terms of their utilities for the same task have been plagued by ambiguities. Researchers of this community are recommended to collaborate on creating large-scale and canonically acceptable benchmark evaluation datasets for various situations and tasks in the DbA process. For example, the recently released FOBIE dataset [16] was developed to support the semi-open relationship extraction for nature-inspired engineering. Such a benchmarking dataset enables the measurement of the effectiveness of novel algorithms against those of other state-of-the-art approaches.

\subsection{Applications}

Based on the four-phase model of making analogies in cognitive science, we assessed the applications of the DbA tools and methods, and categorized each of them into one or more phases accordingly. From Table 2 , it can be distinctly observed that most of them fall within the analogy representation and retrieval subphases, which are the foundations of the entire DbA process. In most studies on these two subphases, text mining-related techniques were adopted to represent and identify candidate analogies for further use. Some researchers have utilized modern deep learning-based AI techniques to construct high-dimensional latent space for design data representation in different forms. These researchers generally posit that, guided by the identified stimuli, human users can continue the mapping and inference process. Although many AI-based methods and tools can recommend the candidate analogy list for users, few can interpret the results such as identifying the type of similarities.

Some studies sought to automate the analogical mapping process, including some systems that predate the 2000 s, most of which are rule-based expert systems, as shown in Fig. 5. Some pre-defined rules, such as syntactic grammar-based rules [81], are embedded within these tools to facilitate automated inference. In addition, some tools implement idea generation by simply combining two ideas within moderate distance for design ideation [17]. Overall, current analogy mapping methods and tools have employed little machine intelligence, which is the major advantage of modern AI techniques. For example, recent deep learning AI models, such as the Siamese network and its variations [104], enable machines to learn pair-wise entity to entity embeddings that encode the specific type of relational information. If we collect a large set of analogy pairs, we may leverage these models to learn the analogical relational representation and conduct the analogical reasoning process.

Regarding the fourth phase, only a few researchers have attempted to evaluate the analogies or ideas generated by the DbA. This may be attributed to, at least, two reasons. First, it is difficult to establish the evaluation criteria. The usefulness and novelty that define "creativeness" may vary according to specific personal needs, organizational contexts, regional cultures, and times [105]. Second, analogy assessment is a domain-related process. A traditional idea or concept in one knowledge domain might be novel in another. Therefore, the space that is newly transformed through analogy reasoning may require novel types of evaluation. A powerful evaluation mechanism would determine the effectiveness and usefulness of new data-driven DbA systems. There is still much room for researchers to develop robust, interpretable, and convincing analogy evaluation methods.

\footnotetext{
${ }^{6}$ https://ddi.sutd.edu.sg/data-driven-design-by-analogy/

${ }^{7}$ https://www.thingiverse.com/
} 
Table 2: The applications of DbA tools and methods 8

\begin{tabular}{|c|c|c|c|c|}
\hline Tools or Methods & $\begin{array}{l}\text { Analogical } \\
\text { Encoding }\end{array}$ & $\begin{array}{c}\text { Analogical } \\
\text { Retrieval }\end{array}$ & $\begin{array}{l}\text { Analogical } \\
\text { Mapping }\end{array}$ & $\begin{array}{l}\text { Analogical } \\
\text { Evaluation }\end{array}$ \\
\hline TRIZ, 1956 [65] & & & $*$ & \\
\hline ARGO, 1988 [58] & * & $*$ & & \\
\hline IDEAL, 1996 [57] & * & * & * & \\
\hline KRITIK, 1997 [56] & $*$ & * & * & \\
\hline BIO-TRIZ, 2002 [67] & & & * & \\
\hline Pat-Analyzer, 2007 [66] & & & * & \\
\hline E2B, 2010 [78], 2011 [77] & & & * & \\
\hline PAnDA, $2011[84]$ & $*$ & * & & \\
\hline WordTree, $2012[4]$ & * & $*$ & & \\
\hline DANE, 2012 [79] & $*$ & $*$ & & \\
\hline IEKG, $2012[\overline{60]}$ & & & * & \\
\hline Fu et al., 2013 [72] & $*$ & & & \\
\hline Func. Vector, 2014 [5] & $*$ & $*[50]$ & & \\
\hline Four-box, 2014 [80] & & & & * \\
\hline Cheong et al., $2014[81]$ & & * & * & \\
\hline Glier et al., $2014[82]$ & & & & * \\
\hline AskNature, 2014 [83] & $*$ & & & \\
\hline DRACULA, 2015 [87] & $*$ & $*$ & * & \\
\hline SEABIRD, 2016 [85] & * & * & * & \\
\hline Analogy Finder, 2016 [68] & & * & & \\
\hline Sanaei et al., 2017 [62] & & $*$ & & \\
\hline Song et al., $2017[41]$ & & * & & \\
\hline B-Link, 2017 [55] & * & $*$ & & $*[19]$ \\
\hline Gilon et al., 2018 [63] & & $*$ & & \\
\hline SOLVENT, 2018 & & * & * & \\
\hline Retriever, 2018 [18] & & * & & \\
\hline Combinator, 2018 [17] & & & * & \\
\hline Idea-Inspire, 2018 [53] & * & * & * & * \\
\hline Kwon et al., 2019 [91] & & $*$ & & \\
\hline TechNet, 2020 [54] & $*[14]$ & $*[70]$ & & $*[19]$ \\
\hline VISION, 2020 [7] & $*$ & $*$ & & \\
\hline Zhang et al., 2020 [92] & * & * & & \\
\hline FOBIE, 2020 [89] & & & * & \\
\hline Jiang et al., 2021 [13] & $*$ & $*$ & & \\
\hline InnoGPS, 2021 [15] & $*$ & & $*$ & \\
\hline
\end{tabular}

\subsection{Methods}

The AI-based techniques that have already been used in DbA studies are summarized in Table 3 As shown in Fig. 5 , the broadly defined NLP methods, including text mining, topic modeling, semantic networks, and pre-trained word embeddings, have played essential roles in supporting all stages of DbA. These methods aim at understanding and mining the textual nuances of natural language. However, some of them tackled the rough source data using only traditional textual pre-processing methods. They developed some syntactic rules for the processed dataset, which were still limited to the human experience. These pre-defined rules cannot be updated automatically to match the rapid advances in AI technologies. Some researchers that adopted knowledge graph techniques generally relied on the commonsense semantic networks trained on non-engineering data sources such as WordNet and ConceptNet. However, engineering designers have perceptions of technical terms that are distinct from the commonsense knowledge [54]. In this case, the technological and engineering semantic networks (e.g., TechNet and B-link) that were recently developed based on engineering data can serve as infrastructure to support broad DbA studies.

\footnotetext{
${ }^{8}$ The star symbol "*" indicates the method/tool itself involved in a specific application. The reference after the star symbol indicates that the method/tool has been utilized in another study for a specific application goal.
} 
Table 3: Existing AI-based methods and algorithms that have been adopted or developed for DbA

\begin{tabular}{|c|c|c|}
\hline Approach & Method & Refs \\
\hline \multirow{4}{*}{$\begin{array}{l}\text { Artificial Neural } \\
\text { Network (ANN) }\end{array}$} & Convolutional Neural Network (CNN) & [13, 92] \\
\hline & Recurrent Neural Network (RNN) & 54 \\
\hline & Bi-directional Recurrent Neural Network (Bi-RNN) & 63, 89 \\
\hline & Long short-term memory (LSTM) & [62] \\
\hline \multirow[b]{2}{*}{ Clustering } & Agglomerative Hierarchical Clustering (AHC) & [41] \\
\hline & Relevance score-based clustering (RSC) & [7, 72] \\
\hline \multirow{3}{*}{ Classification } & Support Vector Machine (SVM) & [62, 82] \\
\hline & Naive Bayes (NB) & [82] \\
\hline & K-Nearest Neighbors (KNN) & [82] \\
\hline \multirow[t]{2}{*}{ Text mining } & Text preprocessing techniques & $\frac{[53,54,55,63,64,66,68,}{81,84,85]}$ \\
\hline & Syntactic Analysis & {$[81,89]$} \\
\hline \multirow{4}{*}{ Word Embedding } & Word2Vec & {$[53,54,64]$} \\
\hline & Doc2Vec & 64 \\
\hline & Glove & [64] \\
\hline & ELMO & [89] \\
\hline \multirow{3}{*}{ Topic-modeling } & Latent Semantic Analysis (LSA) & [72] \\
\hline & Latent Dirichlet Allocation (LDA) & {$[106$} \\
\hline & Non-Negative Matrix Factorization (NMF) & [7] \\
\hline \multirow{5}{*}{$\begin{array}{l}\text { Semantic Network } \\
\text { and Knowledge } \\
\text { Graph }\end{array}$} & WordNet & [4,62] \\
\hline & ConceptNet & [17, [18] \\
\hline & Cyc & {$[63$} \\
\hline & TechNet & {$[14,19,70$} \\
\hline & B-Link & [19] \\
\hline \multirow{3}{*}{$\begin{array}{l}\text { Vector Space Method } \\
\text { (VSM) }\end{array}$} & Functional vector space & [5, 50] \\
\hline & Technology space map & [15] \\
\hline & PA and OA space & 84,85 \\
\hline \multirow[b]{2}{*}{ Probability analysis } & Dijkstra's shortest path algorithm & [55] \\
\hline & Bayesian inference approach & [72] \\
\hline Expert system & Heuristic rules-based strategy & 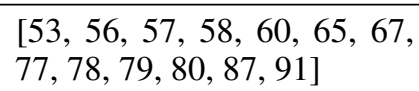 \\
\hline
\end{tabular}

Regarding the other methods, clustering algorithms have been used to represent design data by categories and facilitate the retrieval process [7, 41, 72]. Vector Space Method represents the original data as a vector of subitems, which also benefits both the encoding and retrieval subphases [5, 15, 50, 84, 85]. It is worth mentioning that recent deep learning techniques based on various structure models have shown great potential in some human tasks such as object recognition and classification. Specifically, in DbA studies, some initial intents have been employed to use CNNs and RNNs to support DbA tasks, ranging from analogy representation to analogy mapping [13, 54, 62, 63, 89, 92].

The rapid advances in deep learning-based AI techniques may provide many powerful tools for data-driven $\mathrm{DbA}$. For example, visual and semantic analogy question solving has been explored extensively [104, 107, 108, 109, 110]; majority of the studies involve discovering an extendable mapping from an image and word pair and applying it to another image and word pair to find analogies. For example, Sadeghi et al. [109] utilized a quadruple Siamese network architecture to address the problem associated with creating visual analogies for natural images. Similarly, Rossiello et al. [104] used hierarchical Siamese networks to learn relational representations by textual analogy. Moreover, various deep learning models, besides deep RNNs and CNNs and their variations, generative adversarial networks (GAN) [111], transformer [112] and graph neural networks (GNN) [113], have proven effective for learning complex features from datasets. More recently, the transformer-based models built by Open.AI, such as BERT [114], GPT-3 [115], and DALL.E [116], have exhibited record-breaking performances in understanding and generating new texts and images from given conditions. DbA researchers may adopt these state-of-the-art models from the frontier of AI research to support relational analogy representation and analogical reasoning for engineering design. However, note that the model 
interpretability of deep learning has always been a limiting factor for real-world use cases, where model outputs should be explained. Developing explainable AI (XAI) models [117] for DbA tasks is still challenging for the community currently and needs more exploration.

\section{Future Opportunities and Directions}

In this paper, we have analyzed the literature on data-driven $\mathrm{DbA}$ to elucidate its state-of-the-art. Although existing methods and tools have already shown their ability to support the DbA process, many areas remain under-researched. In this section, we map the course of feasible directions for future research from the perspective of data, methods, applications, and their interactions.

\section{(1) New data sources exploration}

Based on our literature review, patent and scientific paper databases are suitable data sources because of their large size, as well as the rich information they possess on potential design inspiration. Both have been used in many data-driven DbA studies [5, 15, 64]. Although crowdsourced design data [97, 98, 99] and crowdfunded project data [100] have been explored in design research and are also potentially useful for DbA studies, they have not been fully exploited. In addition, most of the current data-driven $\mathrm{DbA}$ methods were designed to merely mine textual information as analogy candidates; only a few researchers have focused on the data of other modalities [13, 92]. Scholars have revealed that designers often prefer visual representations to textual descriptions for idea generation [118]. Therefore, various kinds of visual design sources, such as technical images [13], design sketches [92], 3D product models [101, 102, 103], ought to be exploited and mined. It is recommended that researchers develop more tools based on the new data sources and broad data modalities.

\section{(2) Benchmark datasets construction}

The lack of distinct ground-truth and gold-standard benchmarks in DbA-related problems makes it impossible to directly compare different $\mathrm{DbA}$ methods and evaluate their performance in DbA applications reliably. For example, different analogy retrieval methods are typically applied to different datasets; literature [63] used a scientific paper database and literature [30] used a patent database. In this case, authors often define their own criteria for assessing performance in the absence of ground truth, which makes it difficult to determine the relative performance, strengths, and weaknesses of each method. This also prevents the community from following, adopting, and iterating on the state-of-the-art algorithms. Summers et al. [119] similarly discussed the necessity and benefits of establishing benchmark datasets for function representation in engineering design. As a broader field, data-driven DbA requires a series of benchmark datasets for four different subprocesses. Some attempts have been made to create such benchmarking standard, such as the recently released FOBIE dataset [16] that was developed to support the semi-open relationship extraction for nature-inspired engineering. However, in practice, we should note that the ground truth labels used as the benchmarking standard may suffer from the same biases as the training data. We recommend that researchers create a series of benchmark datasets by combining different data sources for DbA tasks to surmount such limitations.

\section{(3) Engineering knowledge graph-based applications}

Data-driven DbA requires the knowledge database in the backend. When it comes to the large and cross-domain ones, the knowledge base needs to be structured and organized to enable effective retrieval and mapping. In the engineering design literature, the network map of technology domains [40, 41] and semantic networks [54, 55] have been created and used to guide the retrieval of analogical design stimuli based on the quantified knowledge distance between domains [15] or the semantic distance between concepts [70]. Meanwhile, the most frequently used knowledge bases are the large pre-trained commonsense knowledge graphs such as WordNet, ConceptNet and FreeBase [120, 121].

In the field of $\mathrm{AI}$, the knowledge graph notion specifically refers to the qualitative networks composed of many heterogenous <entity, relation, entity> triplets [122, 123]. The relations are qualitatively specific and sensible for humans, in contrast to the one-dimensional semantic distance or knowledge distance constructs. The past decade has seen the rapid adoption of large knowledge graphs in powering knowledge representation, retrieval, and mapping tasks in many AI applications. Google, Facebook, Amazon, TikTok, among others, have developed and used knowledge graphs to support the search, recommendation, and query engines in their AI products and services.

Therefore, it is recommended that researchers extend the uses of such large pre-trained cross-domain semantic networks (e.g., B-Link [55] and TechNet [54]) or knowledge graphs (e.g., WordNet [95] and ConceptNet [96]) as knowledge bases for developing knowledge-based expert systems for DbA. Furthermore, it will be a promising effort to develop graph knowledge bases in which the entities are presented at the hierarchical domain to concept levels and both the quantitative and qualitative relations between entities are pre-trained, to augment analogy retrieval and mapping across domains and concepts. 


\section{(4) Deep learning solutions, new models, architectures, and XAI}

Deep learning techniques based on various structure models, such as CNNs and RNNs, have shown their strengths in supporting DbA tasks, ranging from analogy representation to analogy mapping [13, 54, 63, 89, 92, 97]. On the basis of these efforts, the up-to-date basic deep learning structures (e.g., transformer [112] and GNN [113]) and large-scale pre-trained models (e.g., BERT [114] and GPT [115]) ought to be explored and utilized to facilitate all DbA subprocesses. There exist many opportunities for collaborative research among the psychological cognition, engineering design, and deep learning communities, and new models and algorithms can be developed to handle various types of data sources. Specifically, in analogy mapping and evaluation tasks, domain expertise is required to interpret the results yielded by deep learning algorithms.

In addition, understanding the fundamental properties and interpretability of deep learning is an essential part of such data-driven studies. For example, when we use deep learning to retrieve analogy candidates, being aware of the similarity type of analogies is as important as obtaining the recommended analogy candidate list. The retrieval systems should provide users more insightful information with the final outputs. In this regard, one of the pioneer works is SOLVENT [64], which attempts to interpret the types of analogies by annotating and mapping different aspects of research papers. Beyond this, researchers may combine the modern deep learning with traditional interpretable machine learning techniques (e.g., decision trees and Bayesian classifiers) to develop more explainable models for DbA.

\section{(5) New subprocess of data-driven DbA: Analogy-based design synthesis}

In the traditional $\mathrm{DbA}$ process, following the analogy retrieval and mapping steps, the final design synthesis stage typically requires the experience, intelligence, and efforts of designers. As a comparison, various large-scale datasets collected and represented for previous DbA subprocesses also provide opportunities for scholars to explore the deep learning-based data synthesis and generative methods, which we term as a new subprocess of data-driven DbA, analogy-based design synthesis. Unlike traditional design synthesis strategies, such as shape grammars and constraint programming, data-driven design synthesis methods do not necessarily require expert knowledge and can automatically learn to generate plausible new designs from datasets [124, 125, 126, 127, 128, 129, 130].

Among various data-driven design synthesis methods, recent deep generative models have gained significant attention because of their ability to learn complex features from samples. GAN [111] and Variational autoencoder (VAE) [131] are the most commonly used deep generative models to assist engineering design. Specifically, variations of GAN have been used in designing airfoils [124, 125], car wheels [126], bicycles [127], airplane [129] and social robots [130], and VAE has been used in designing material microstructures [128]. Recently, the pre-trained GPT [115] and DALL.E [116] models released by Open.AI have exhibited record-breaking performances in creating novel texts and images. As these synthesis methods typically require given inputs as the initial design information, the identified analogies from the previous $\mathrm{DbA}$ steps may potentially guide the direction of design generation. Therefore, it is recommended that researchers extend the use of these existing AI-based generative models and algorithms to develop analogy-based design synthesis tools.

Taken together, the foregoing propositions can be merged and implemented in one future data-driven DbA system that applies various latest AI techniques to different DbA subprocesses. In Fig. 6, we conceptualized such a data-driven DbA system, in comparison with the traditional one. In this future system, multi-source and multimodal design-related data are embedded and stored in a high-dimensional vector space through representation learning. The vector space is dynamic and can be updated based on the requirements and performance of the downstream analogical tasks. Analogical retrieval, mapping and evaluation will be conducted based on the distributed representation of design data samples through various data mining techniques. Benchmark datasets are available for researchers to make comparisons among new and alternative algorithms. During analogical reasoning, interpretable machine learning will enable us peek into the support evidence of analogy making, such as providing similarity types among analogies. Finally, deep generative models will be utilized to automatically generate new designs according to the recommended analogy pairs and their mapping relationship. In this system, human-computer interaction may play an essential role in this process to improve the quality of design synthesis. These newly created designs can also be assessed by the intelligent evaluation model that considers user needs, sentiments, and compassion.

Furthermore, it is important to note that the design of future data-driven intelligent $\mathrm{DbA}$ systems should be grounded on the scientific understanding of analogy. The available cognitive science foundations of analogy had been mainly developed via traditional and small human experiments and remained abstract and at a macro-level of understanding and application [3, 22, 26, 27, 29, 38, 39, 46, 76]. With the growing development and uses of data-driven DbA systems, we anticipate a growing amount of data on various DbA processes and outcomes to be generated. Such big data may offer new research opportunities by analyzing them to nuance and advance our scientific understanding of the cognitive process and mechanisms of analogy. In turn, the new understanding can be applied to develop more explainable AI systems for design by analogy. 


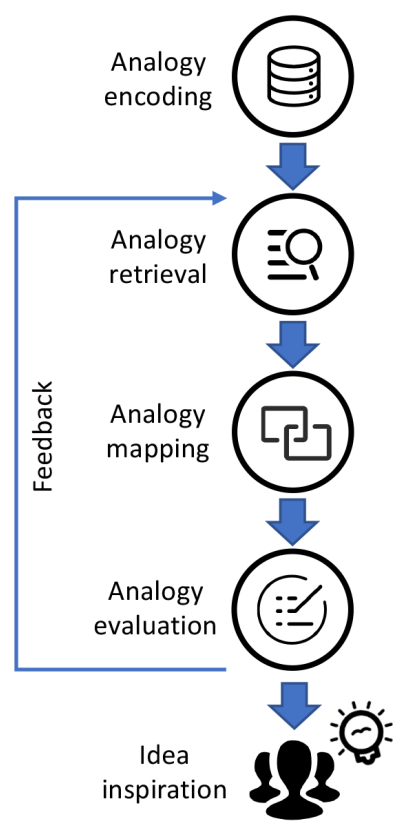

a. Traditional DbA process

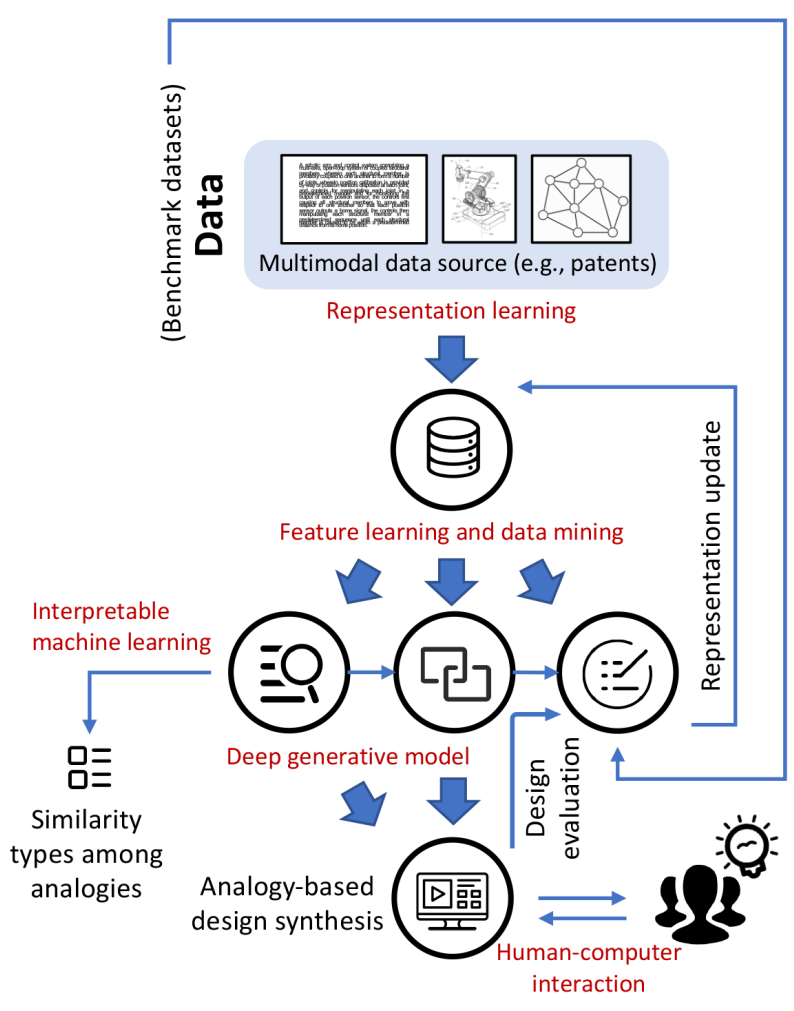

b. Future data-driven DbA system

Figure 6: Schematic comparison between the traditional DbA process and future data-driven DbA system

\section{Conclusion}

Data-driven Design-by-Analogy presents great potential to augment design creativity and enhance innovation. We contribute to the literature by elucidating the state-of-the-art data-driven DbA studies and identifying new and feasible future research opportunities. Our structured review of the data, applications, and methods of existing data-driven DbA systems and tools provides a foundation or basis for researchers to follow the relevant latest efforts in a coherent form. Several research directions are proposed in terms of data, applications, and methods, respectively, to bridge the gap between engineering design and AI research. We hope that our review and propositions may guide the efforts of researchers aiming to develop more powerful and intelligent data-driven DbA methods, tools, and systems.

In this paper, although we only focus on data-driven $\mathrm{DbA}$ methods and tools, AI and data science techniques can be also useful to augment other classical engineering design methods [132], such as TRIZ [65, 66], design heuristics [133, 134], design principles [94, 135], design structure matrix [136, 137], product family and platform design [138, 139], first principles [140, 141, 142], C-K [143], blending [144] and combinational design [99, 145]. We hope that researchers of these relevant fields may also find inspirations from this paper to advance the data-driven approaches for their design methodologies with latest AI and data science technologies.

\section{Acknowledgements}

The authors acknowledge the funding support for this work received from the SUTD-MIT International Design Center and SUTD Data-Driven Innovation Laboratory (DDI, https://ddi.sutd.edu.sg/), National Natural Science Foundation of China (52035007, 51975360), Special Program for Innovation Method of the Ministry of Science and Technology, China (2018IM020100), National Social Science Foundation of China (17ZDA020). The support from CU Denver's College of Engineering, Design and Computing (CEDC, https://engineering.ucdenver.edu/) and the Comcast Media 
and Technology Center (CMTC, https://comcastmediatechcenter.org/) are also acknowledged. Any ideas, results and conclusions contained in this work are those of the authors, and do not reflect the views of the sponsors.

\section{References}

[1] David G Ullman. The mechanical design process, volume 2. McGraw-Hill New York, 1992.

[2] Ashok K Goel. Design, analogy, and creativity. IEEE expert, 12(3):62-70, 1997.

[3] Bo T Christensen and Christian D Schunn. The relationship of analogical distance to analogical function and preinventive structure: The case of engineering design. Memory \& cognition, 35(1):29-38, 2007.

[4] J S Linsey, A B Markman, and K L Wood. Design by analogy: A study of the WordTree method for problem re-representation. ASME Journal of Mechanical Design, 134(4):041009, 2012.

[5] Jeremy Murphy, Katherine Fu, Kevin Otto, Maria Yang, Dan Jensen, and Kristin Wood. Function based designby-analogy: a functional vector approach to analogical search. ASME Journal of Mechanical Design, 136(10): $101102,2014$.

[6] Hyeonik Song and Katherine Fu. Design-by-Analogy: Exploring for Analogical Inspiration With Behavior, Material, and Component-Based Structural Representation of Patent Databases. ASME Journal of Computing and Information Science in Engineering, 19(2):021014, 2019.

[7] Hyeonik Song, Jacob Evans, and Katherine Fu. An exploration-based approach to computationally supported design-by-analogy using D3. Artificial Intelligence for Engineering Design, Analysis and Manufacturing, 34(4): 444-457, 2020.

[8] Ashok K Goel and L H Shu. Analogical thinking: An introduction in the context of design. Artificial Intelligence for Engineering Design, Analysis and Manufacturing, 29(2, SI):133-134, 2015.

[9] Julie S Linsey, Ian Tseng, Katherine Fu, Jonathan Cagan, Kristin L Wood, and C Schunn. A Study of Design Fixation, Its Mitigation and Perception in Engineering Design Faculty. ASME Journal of Mechanical Design, 132(4):041003, 2010.

[10] Katherine Fu, Joel Chan, Jonathan Cagan, Kenneth Kotovsky, Christian Schunn, and Kristin Wood. The meaning of "near" and "far": the impact of structuring design databases and the effect of distance of analogy on design output. ASME Journal of Mechanical Design, 135(2):021007, 2013.

[11] Carlye Lauff, Yu Hui Wee, Kenneth Teo, Sabrina Png, Amanda Swee, and Kristin L. Wood. Design Innovation (DI) Methodology Handbook. Singapore University of Technology and Design (SUTD) and University of Colorado Denver (CU Denver) I Anschutz Medical Campus, Singapore, 2021. ISBN 978-981-18-1207-1.

[12] Suzana Linic, Vojkan Lucanin, Srdjan Zivkovic, Marko Rakovic, and Mirjana Puharic. Experimental and Numerical Methods for Concept Design and Flow Transition Prediction on the Example of the Bionic HighSpeed Train. In Proceedings of the International Conference of Experimental and Numerical Investigations and New Technologies, pages 65-82. Springer, 2020.

[13] Shuo Jiang, Jianxi Luo, Guillermo Ruiz-pava, Jie Hu, and Christopher L Magee. Deriving design feature vectors for patent images using convolutional neural networks. ASME Journal of Mechanical Design, 143(6):061405, 2021.

[14] Serhad Sarica and Jianxi Luo. Design Knowledge Representation with Technology Semantic Network. In Proceedings of the Design Society: International Conference on Engineering Design (ICED), Gothenburg, Sweden, Aug. 16-20, 2021.

[15] Jianxi Luo, Serhad Sarica, and Kristin L. Wood. Guiding Data-Driven Design Ideation by Knowledge Distance. Knowledge-Based Systems, 218:106873, 2021.

[16] Ruben Kruiper, Julian Vincent, Jessica Chen-Burger, Marc Desmulliez, and Ioannis Konstas. In Layman's Terms: Semi-Open Relation Extraction from Scientific Texts. In Annual Meeting of the Association for Computational Linguistics (ACL), pages 1489-1500, 2020.

[17] Ji Han, Feng Shi, Liuqing Chen, and Peter R N Childs. The Combinator: a computer-based tool for creative idea generation based on a simulation approach. Design Science, 4(e11):1-34, 2018.

[18] Ji Han, Feng Shi, Liuqing Chen, and Peter R N Childs. A computational tool for creative idea generation based on analogical reasoning and ontology. Artificial Intelligence for Engineering Design, Analysis and Manufacturing, 32(4):462-477, 2018. 
[19] Ji Han, Hannah Forbes, Feng Shi, Jia Hao, and Dirk Schaefer. A Data-Driven Approach for Creative Concept Generation and Evaluation. In Proceedings of the Design Society: DESIGN Conference, volume 1, pages 167-176, Online, 2020.

[20] Brian H Ross. This is like that: The use of earlier problems and the separation of similarity effects. Journal of Experimental Psychology: Learning, Memory, and Cognition, 13(4):629, 1987.

[21] Arthur B Markman. Constraints Analogical on Inference. Cognitive science, 21(4):373-418, 1997.

[22] Arthur B Markman and Dedre Gentner. Structural alignment during similarity comparisons. Cognitive psychology, 25(4):431-467, 1993.

[23] Boicho Kokinov and Robert M French. Computational models of analogy-making. Encyclopedia of cognitive science, 1:113-118, 2003.

[24] Rogers P Hall. Computational approaches to analogical reasoning: A comparative analysis. Artificial intelligence, 39(1):39-120, 1989.

[25] Michael J French. Conceptual design for engineers. Springer, 1985.

[26] Arthur B Markman, Kristin L Wood, Julie S Linsey, Jeremy T Murphy, and Jeffrey Laux. Supporting innovation by promoting analogical reasoning. Tools for innovation, 1(9):85-104, 2009.

[27] Dedre Gentner and Linsey A Smith. Analogical Learning and Reasoning. In The Oxford handbook of cognitive psychology, pages 668-681. Oxford University Press, 2013.

[28] Thomas B Ward and Yuliya Kolomyts. Cognition and creativity. The Cambridge handbook of creativity, pages 93-112, 2010.

[29] Jonathan Hey, Julie Linsey, Alice M Agogino, and Kristin L Wood. Analogies and metaphors in creative design. International Journal of Engineering Education, 24(2):283-294, 2008.

[30] Paul-armand Verhaegen, D Joris, Dennis Vandevenne, Simon Dewulf, and Joost R Duflou. Identifying candidates for design-by-analogy. Computers in Industry, 62(4):446-459, 2011. ISSN 0166-3615.

[31] Dedre Gentner and Arthur B Markman. Structure mapping in analogy and similarity. American psychologist, 52 (1):45-56, 1997.

[32] David J Chalmers, Robert M French, and Douglas R Hofstadter. High-level perception, representation, and analogy: A critique of artificial intelligence methodology. Journal of Experimental \& Theoretical Artificial Intelligence, 4(3):185-211, 1992.

[33] Thomas G Evans. A program for the solution of a class of geometric-analogy intelligence-test questions. In Semantic Information Processing, pages 271-353. MIT Press, 1964.

[34] John E Hummel and Keith J Holyoak. Distributed representations of structure: A theory of analogical access and mapping. Psychological review, 104(3):427, 1997.

[35] Boicho Kokinov and Alexander Petrov. Dynamic extension of episode representation in analogy-making in AMBR. In Proceedings of the 22nd Annual Conference of the Cognitive Science Society (CogSci), pages 274-279, 2000.

[36] Kazjon Grace, John Gero, and Rob Saunders. Interpretation-driven mapping: A framework for conducting search and rerepresentation in parallel for computational analogy in design. Artificial Intelligence for Engineering Design, Analysis and Manufacturing, 29(2, SI):185-201, 2015.

[37] Robert M French. The computational modeling of analogy-making. Trend in cognitive sciences, 6(5):200-205, 2002.

[38] Amaninder Singh Gill, Arnold N Tsoka, and Chiradeep Sen. Dimensions of Product Similarity in Design by Analogy: An Exploratory Study. In Proceedings of the 2019 ASME International Design Engineering Technical Conferences and Computers and Information in Engineering Conference (IDETC/CIE), page V007T06A010, Anaheim, California, USA, Aug.18-21, 2019.

[39] Arnold N Tsoka, Jicmat Ali Tribaldos, and Chiradeep Sen. Dimensions of Similarity Used to Identify Products As Sources of Analogy. In Proceedings of the 2020 ASME International Design Engineering Technical Conferences and Computers and Information in Engineering Conference (IDETC/CIE), page V008T08A009, Virtual, Online, Aug. 17-19, 2020.

[40] V Srinivasan, Binyang Song, Jianxi Luo, Karupppasamy Subburaj, Mohan Rajesh Elara, Lucienne Blessing, and Kristin Wood. Does analogical distance affect performance of ideation? ASME Journal of Mechanical Design, 140(7):071101, 2018. 
[41] Binyang Song, V Srinivasan, and Jianxi Luo. Patent stimuli search and its influence on ideation outcomes. Design Science, 3(e25):1-25, 2017.

[42] Joel Chan, Katherine Fu, Christian Schunn, Jonathan Cagan, Kristin Wood, and Kenneth Kotovsky. On the benefits and pitfalls of analogies for innovative design: Ideation performance based on analogical distance, commonness, and modality of examples. ASME Journal of mechanical design, 133(8):081004, 2011.

[43] Ivey Chiu and Li H Shu. Investigating effects of oppositely related semantic stimuli on design concept creativity. Journal of Engineering Design, 23(4):271-296, 2012.

[44] Ross A Malaga. The effect of stimulus modes and associative distance in individual creativity support systems. Decision Support Systems, 29(2):125-141, 2000.

[45] Ellen Enkel and Oliver Gassmann. Creative imitation: exploring the case of cross-industry innovation. $R \& D$ Management, 40(3):256-270, 2010.

[46] Julie S Linsey, Kristin L Wood, and Arthur B Markman. Modality and representation in analogy. Artificial Intelligence for Engineering Design, Analysis and Manufacturing, 22(2):85-100, 2008.

[47] Olufunmilola Atilola and Julie Linsey. Representing analogies to influence fixation and creativity: A study comparing computer-aided design, photographs, and sketches. Artificial Intelligence for Engineering Design, Analysis and Manufacturing, 29(2, SI):161-171, 2015.

[48] Hyeon Ik Song, Ricardo Lopez, Katherine Fu, and Julie Linsey. Characterizing the Effects of Multiple Analogs and Extraneous Information for Novice Designers in Design-by-Analogy. ASME Journal of Mechanical Design, 140(3):031101, 2018.

[49] Ian Tseng, Jarrod Moss, Jonathan Cagan, and Kenneth Kotovsky. The role of timing and analogical similarity in the stimulation of idea generation in design. Design Studies, 29(3):203-221, 2008.

[50] Katherine Fu, Jeremy Murphy, Maria Yang, Kevin Otto, Dan Jensen, and Kristin Wood. Design-by-analogy: experimental evaluation of a functional analogy search methodology for concept generation improvement. Research in Engineering Design, 26(1):77-95, 2015.

[51] Diana P Moreno, Lucienne T Blessing, Maria C Yang, Alberto A Hernandez, and Kristin L Wood. Overcoming design fixation: Design by analogy studies and nonintuitive findings. Artificial Intelligence for Engineering Design, Analysis and Manufacturing, 30(2, SI):185-199, 2016.

[52] Thomas B Ward. Analogical distance and purpose in creative thought: Mental leaps versus mental hops. Advances in analogy research: Integration of theory and data from the cognitive, computational, and neural sciences, pages 221-230, 1998.

[53] L Siddharth and Amaresh Chakrabarti. Evaluating the impact of Idea-Inspire 4.0 on analogical transfer of concepts. Artificial Intelligence for Engineering Design, Analysis and Manufacturing, 32(4):431-448, 2018.

[54] Serhad Sarica, Jianxi Luo, and Kristin L Wood. TechNet: Technology semantic network based on patent data. Expert Systems with Applications, 142:112995, 2020.

[55] Feng Shi, Liuqing Chen, Ji Han, and Peter Childs. A data-driven text mining and semantic network analysis for design information retrieval. ASME Journal of Mechanical Design, 139(11):111402, 2017.

[56] Ashok Goel, Sambasiva Bhatta, and Eleni Stroulia. Kritik: An early case-based design system. In Issues and applications of case-based reasoning in design, pages 87-132. MIT Press, 1997.

[57] Sambasiva R Bhatta, Ashok K Goel, and Others. From design experiences to generic mechanisms: model-based learning in analogical design. Artificial Intelligence for Engineering Design, Analysis and Manufacturing, 10(2): $131-136,1996$.

[58] Michael N Huhns and Ramon D Acosta. Argo: a system for design by analogy. In The 4th Conference on Artificial Intelligence Applications, pages 146-151, San Diego, CA, USA, 1988.

[59] Lena Qian, John S Gero, and Others. Function-behavior-structure paths and their role in analogy-based design. Artificial Intelligence for Engineering Design, Analysis and Manufacturing, 10(4):289-312, 1996.

[60] Yoram Reich and Offer Shai. The interdisciplinary engineering knowledge genome. Research in Engineering Design, 23(3):251-264, 2012.

[61] Robert B Stone and Kristin L Wood. Development of a Functional Basis for Design. ASME Journal of Mechanical Design, 122(4):359-370, 2000.

[62] Roozbeh Sanaei, Wei Lu, Lucinne T.M. Blessing, Kevin N. Otto, and Kristin L. Wood. Analogy retrieval through textual inference. In Proceedings of the 2017 ASME International Design Engineering Technical Conferences and Computers and Information in Engineering Conference (IDETC/CIE), page V02AT03A007, Cleveland, USA, Aug. 6-9, 2017. 
[63] Karni Gilon, Joel Chan, Felicia Y Ng, Hila Liifshitz-Assaf, Aniket Kittur, and Dafna Shahaf. Analogy mining for specific design needs. In Proceedings of the 2018 CHI Conference on Human Factors in Computing Systems, Montréal, QC, Canada, Apr. 21-26, 2018.

[64] Joel Chan, Joseph Chee Chang, Tom Hope, Dafna Shahaf, and Aniket Kittur. SOLVENT : A Mixed Initiative System for Finding Analogies between Research Papers. In Proceedings of the ACM on Human-Computer Interaction, pages 1-21, New York, USA, 2018.

[65] Genrikh Saulovich Altshuller. The innovation algorithm: TRIZ, systematic innovation and technical creativity. Technical innovation center Inc., 1999.

[66] Gaetano Cascini and Davide Russo. Computer-aided analysis of patents and search for TRIZ contradictions. International Journal of Product Development, 4(1):52-67, 2007.

[67] Julian F V Vincent and Darrell L Mann. Systematic technology transfer from biology to engineering. Philosophical Transactions of the Royal Society of London. Series A: Mathematical, Physical and Engineering Sciences, 360(1791):159-173, 2002.

[68] Anthony Mccaffrey and West Brookfield. Analogy Finder, 2016, US Patent.

[69] Serhad Sarica, Binyang Song, En Low, and Jianxi Luo. Engineering knowledge graph for keyword discovery in patent search. In Proceedings of the Design Society: International Conference on Engineering Design (ICED), The Netherlands, Aug. 5-8, 2019.

[70] Serhad Sarica, Binyang Song, Jianxi Luo, and Kristin L. Wood. Idea Generation with Technology Semantic Network. Artificial Intelligence for Engineering Design, Analysis and Manufacturing, pages 1-19, 2021.

[71] Liuqing Chen, Pan Wang, Hao Dong, Feng Shi, Ji Han, Yike Guo, Peter R.N. Childs, Jun Xiao, and Chao Wu. An artificial intelligence based data-driven approach for design. Journal of Visual Communication and Image Representation, 61:10-22, 2019.

[72] Katherine Fu, Jonathan Cagan, Kenneth Kotovsky, and Kristin Wood. Discovering structure in design databases through functional and surface based mapping. ASME Journal of Mechanical Design, 135(3):031006, 2013.

[73] Julian F V Vincent, Olga A Bogatyreva, Nikolaj R Bogatyrev, Adrian Bowyer, and Anja-Karina Pahl. Biomimetics: its practice and theory. Journal of the Royal Society Interface, 3(9):471-482, 2006.

[74] Amaresh Chakrabarti and L H Shu. Biologically inspired design. Artificial Intelligence for Engineering Design Analysis and Manufacturing, 24(4):453-454, 2010.

[75] Jacquelyn K S Nagel and Robert B Stone. A computational approach to biologically inspired design. Artificial Intelligence for Engineering Design Analysis and Manufacturing, 26(2, SI):161-176, 2012.

[76] Katherine Fu, Diana Moreno, Maria Yang, and Kristin L Wood. Bio-Inspired Design: An Overview Investigating Open Questions From the Broader Field of Design-by-Analogy. ASME Journal of Mechanical Design, 136(11, SI):111102, 2014.

[77] H Cheong, I Chiu, L H Shu, R B Stone, and D A McAdams. Biologically Meaningful Keywords for Functional Terms of the Functional Basis. ASME Journal of Mechanical Design, 133(2):021007, 2011.

[78] Jacquelyn K S Nagel, Robert B Stone, and Daniel A McAdams. An Engineering-to-Biology Thesaurus for Engineering Design. In Proceedings of the 2010 ASME International Design Engineering Technical Conferences and Computers and Information in Engineering Conference (IDETC/CIE), pages 117-128, Montreal, Quebec, Canada, Aug. 15-18, 2010.

[79] Swaroop Vattam, Bryan Wiltgen, Michael Helms, Ashok K Goel, and Jeannette Yen. DANE: fostering creativity in and through biologically inspired design. In The proceedings of 1st International Conference on Design Creativity (ICDC), pages 115-122. The Design Society, Kobe, Japan, Nov. 29-Dec. 1, 2010.

[80] Michael Helms and Ashok K Goel. The Four-Box Method: Problem Formulation and Analogy Evaluation in Biologically Inspired Design. ASME Journal of Mechanical Design, 136(11, SI):111106, 2014.

[81] Hyunmin Cheong and L H Shu. Retrieving Causally Related Functions From Natural-Language Text for Biomimetic Design. ASME Journal of Mechanical Design, 136(8):081008, 2014.

[82] Michael W Glier, Daniel A McAdams, and Julie S Linsey. Exploring Automated Text Classification to Improve Keyword Corpus Search Results for Bioinspired Design. ASME Journal of Mechanical Design, 136(11, SI): $111103,2014$.

[83] Jon-Michael Deldin and Megan Schuknecht. The AskNature database: enabling solutions in biomimetic design. In Biologically inspired design, pages 17-27. Springer, 2014. 
[84] Paul-Armand Verhaegen, Jef Peeters, Dennis Vandevenne, Simon Dewulf, and Joost R Duflou. Effectiveness of the PAnDA ideation tool. Procedia engineering, 9:63-76, 2011.

[85] Dennis Vandevenne, Paul-Armand Verhaegen, Simon Dewulf, and Joost R Duflou. SEABIRD: Scalable search for systematic biologically inspired design. Artificial Intelligence for Engineering Design, Analysis and Manufacturing, 30(1):78-95, 2016.

[86] Briana Lucero, Vimal K Viswanathan, Julie S Linsey, and Cameron J Turner. Identifying Critical Functions for Use Across Engineering Design Domains. ASME Journal of Mechanical Design, 136(12):121101, 2014.

[87] Briana Lucero, Cameron J Turner, and Julie Linsey. Design Repository and Analogy Computation via Unit Language Analysis (DRACULA) Repository Development. In Proceedings of the 2015 ASME International Design Engineering Technical Conferences and Computers and Information in Engineering Conference (IDETC/CIE), page V01AT02A014, Boston, USA, Aug. 2-5, 2015.

[88] Amaresh Chakrabarti, Prabir Sarkar, B Leelavathamma, and B S Nataraju. A functional representation for aiding biomimetic and artificial inspiration of new ideas. Artificial Intelligence for Engineering Design, Analysis and Manufacturing, 19(2):113-132, 2005.

[89] Ruben Kruiper, Julian F V Vincent, Jessica Chen-Burger, Marc P Y Desmulliez, and Ioannis Konstas. A Scientific Information Extraction Dataset for Nature Inspired Engineering. In Proceedings of the 12th Language Resources and Evaluation Conference, pages 2078-2085, Marseille, France, May 13-15, 2020.

[90] Devesh Bhasin, Daniel A McAdams, and Astrid Layton. A product architecture-based tool for bioinspired function-sharing. ASME Journal of Mechanical Design, 143(8):814101, 2021.

[91] E Kwon, A Pehlken, K-D. Thoben, A Bazylak, and L H Shu. Visual Similarity to Aid Alternative-Use Concept Generation for Retired Wind-Turbine Blades. ASME Journal of Mechanical Design, 141(3):031116, 2019.

[92] Zijian Zhang and Yan Jin. An Unsupervised Deep Learning Model to Discover Visual Similarity Between Sketches for Visual Analogy Support. In Proceedings of the 2020 ASME International Design Engineering Technical Conferences and Computers and Information in Engineering Conference (IDETC/CIE), page V008T08A003, Virtual, Online, Aug. 17-19, 2020.

[93] Ashok K Goel, Gongbo Zhang, Bryan Wiltgen, Yuqi Zhang, Swaroop Vattam, and Jeannette Yen. On the benefits of digital libraries of case studies of analogical design: Documentation, access, analysis, and learning. Artificial Intelligence for Engineering Design, Analysis and Manufacturing, 29(2, SI):215-227, 2015.

[94] Vikramjit Singh, Stewart M Skiles, Jarden E Krager, Kristin L Wood, Dan Jensen, and Robert Sierakowski. Innovations in Design Through Transformation: A Fundamental Study of Transformation Principles. ASME Journal of Mechanical Design, 131(8):081010, 2009.

[95] George A Miller. WordNet: a lexical database for English. Communications of the ACM, 38(11):39-41, 1995.

[96] Robyn Speer, Joshua Chin, and Catherine Havasi. Conceptnet 5.5: An open multilingual graph of general knowledge. In Proceedings of the 31st AAAI Conference on Artificial IntelligenceFebruary, pages 4444-4451, San Francisco, California, USA, Feb. 4-9, 2017.

[97] Aniket Kittur, Lixiu Yu, Tom Hope, Joel Chan, Hila Lifshitz-Assaf, Karni Gilon, Felicia Ng, Robert E Kraut, and Dafna Shahaf. Scaling up analogical innovation with crowds and AI. Proceedings of the National Academy of Sciences, 116(6):1870-1877, 2019.

[98] Kosa Goucher-Lambert and Jonathan Cagan. Crowdsourcing inspiration: Using crowd generated inspirational stimuli to support designer ideation. Design Studies, 61:1-29, 2019.

[99] Yuejun He, Bradley Camburn, Haowen Liu, Jianxi Luo, Maria Yang, and Kristin Wood. Mining and Representing the Concept Space of Existing Ideas for Directed Ideation. Journal of Mechanical Design, 141(12), 2019.

[100] Chaoyang Song, Jianxi Luo, Katja Hölttä-Otto, Warren Seering, and Kevin Otto. Crowdfunding for Design Innovation: Prediction Model With Critical Factors. IEEE Transactions on Engineering Management, pages $1-12,2020$.

[101] Angel X Chang, Thomas Funkhouser, Leonidas Guibas, Pat Hanrahan, Qixing Huang, Zimo Li, Silvio Savarese, Manolis Savva, Shuran Song, Hao Su, and Others. Shapenet: An information-rich 3d model repository. https://arxiv.org/abs/1512.03012, 2015.

[102] Joseph J Lim, Hamed Pirsiavash, and Antonio Torralba. Parsing Ikea Objects: Fine Pose Estimation. In IEEE International Conference on Computer Vision (ICCV), pages 2992-2999, Sydney, NSW, Australia, Dec.1-8, 2013. 
[103] Sebastian Koch, Albert Matveev, Zhongshi Jiang, Francis Williams, Alexey Artemov, Evgeny Burnaev, Marc Alexa, Denis Zorin, and Daniele Panozzo. Abc: A big cad model dataset for geometric deep learning. In IEEE Conference on Computer Vision and Pattern Recognition (CVPR), pages 9601-9611, Long Beach, CA, USA, June 16-20, 2019.

[104] Gaetano Rossiello, Alfio Gliozzo, Robert Farrell, Nicolas Fauceglia, and Michael Glass. Learning relational representations by analogy using hierarchical siamese networks. In Proceedings of the 2019 Conference of the North American Chapter of the Association for Computational Linguistics: Human Language Technologies (NAACL-HLT), volume 1, pages 3235-3245, Minneapolis, MN, USA, June 2-7, 2019.

[105] Margaret A Boden. Creativity and artificial intelligence. Artificial Intelligence, 103:347-356, 1998.

[106] Kosa Goucher-Lambert, Joshua T Gyory, Kenneth Kotovsky, and Jonathan Cagan. Adaptive Inspirational Design Stimuli: Using Design Output to Computationally Search for Stimuli That Impact Concept Generation. ASME Journal of Mechanical Design, 142(9):091401, 2020.

[107] Fereshteh Sadeghi, C. Lawrence Zitnick, and Ali Farhadi. Deep Visual Analogy-Making. In Proceedings of the 29th Conference on Neural Information Processing Systems (NIPS), pages 1882-1890, Montréal, QC, Canada, Dec. 7-12, 2015.

[108] Hongjing Lu, Ying Nian Wu, and Keith J Holyoak. Emergence of analogy from relation learning. Proceedings of the National Academy of Sciences, 116(10):4176-4181, 2019.

[109] Fereshteh Sadeghi, C Lawrence Zitnick, and Ali Farhadi. VISALOGY: Answering visual analogy questions. In Proceedings of the 29th Conference on Neural Information Processing Systems (NIPS), pages 1882-1890, Montréal, QC, Canada, Dec. 7-12, 2015.

[110] Jing Liao, Yuan Yao, Lu Yuan, Gang Hua, and Sing Bing Kang. Visual attribute transfer through deep image analogy. ACM Transactions on Graphics, 36(4):1-15, 2017.

[111] Ian Goodfellow, Jean Pouget-Abadie, Mehdi Mirza, Bing Xu, David Warde-Farley, Sherjil Ozair, Aaron Courville, and Yoshua Bengio. Generative Adversarial Nets. In Proceedings of the 27th Conference on Neural Information Processing Systems (NIPS), volume 27, pages 2672-2680, Montréal, QC, Canada, Feb. 8-13, 2014.

[112] Ashish Vaswani, Noam Shazeer, Niki Parmar, Jakob Uszkoreit, Llion Jones, Aidan N Gomez, Łukasz Kaiser, and Illia Polosukhin. Attention is all you need. In Proceedings of the 31st Conference on Neural Information Processing Systems (NIPS), pages 5998-6008, Long Beach, CA, USA, Dec. 4-9, 2017.

[113] Zonghan Wu, Shirui Pan, Fengwen Chen, Guodong Long, Chengqi Zhang, and S Yu Philip. A comprehensive survey on graph neural networks. IEEE transactions on neural networks and learning systems, 32(1):4-24, 2020.

[114] Jacob Devlin, Ming-Wei Chang, Kenton Lee, and Kristina Toutanova. Bert: Pre-training of deep bidirectional transformers for language understanding. Proceedings of the 2019 Conference of the North American Chapter of the Association for Computational Linguistics: Human Language Technologies (NAACL-HLT), pages 4171-4186, 2019.

[115] Tom B Brown, Benjamin Mann, Nick Ryder, Melanie Subbiah, Jared Kaplan, Prafulla Dhariwal, Arvind Neelakantan, Pranav Shyam, Girish Sastry, Amanda Askell, and Others. Language models are few-shot learners. The proceedings of 33th Conference on Neural Information Processing Systems (NeurIPS), pages 1877-1901, 2020.

[116] Aditya Ramesh, Mikhail Pavlov, Gabriel Goh, Scott Gray, Chelsea Voss, Alec Radford, Mark Chen, and Ilya Sutskever. Zero-Shot Text-to-Image Generation. https://arxiv.org/abs/2102.12092, 2020.

[117] Alejandro Barredo Arrieta, Natalia Díaz-Rodríguezb, Javier Del Ser, Adrien Bennetot, Siham Tabik, Alberto Barbado, Salvador Garcia, Sergio Gil-López, Daniel Molina, Richard Benjamins, and Others. Explainable Artificial Intelligence (XAI): Concepts, taxonomies, opportunities and challenges toward responsible AI. Information Fusion, 58:82-115, 2020.

[118] Julie S Linsey, Emily F Clauss, Tolga Kurtoglu, Jeremy T Murphy, Kristin L Wood, and Arthur B Markman. An experimental study of group idea generation techniques: understanding the roles of idea representation and viewing methods. ASME Journal of Mechanical Design, 133(3):031008, 2011.

[119] Joshua D Summers, Claudia Eckert, and Ashok K Goel. Function in engineering: Benchmarking representations and models. Artificial Intelligence for Engineering Design, Analysis and Manufacturing, 31(4, SI):401-412, 2017.

[120] Ji Han, Serhad Sarica, Feng Shi, and Jianxi Luo. Semantic Networks for Engineering Design : A Survey. In Proceedings of the Design Society: International Conference on Engineering Design (ICED), Gothenburg, Sweden, Aug. 16-20, 2021. 
[121] Bradley Camburn, Yuejun He, Sujithra Raviselvam, Jianxi Luo, and Kristin Wood. Machine learning-based design concept evaluation. Journal of Mechanical Design, 142(3), 2020.

[122] Amrapali Zaveri, Anisa Rula, Andrea Maurino, Ricardo Pietrobon, Jens Lehmann, and Soeren Auer. Quality assessment for linked data: A survey. Semantic Web, 7(1):63-93, 2016.

[123] Heiko Paulheim. Knowledge graph refinement: A survey of approaches and evaluation methods. Semantic web, 8(3):489-508, 2017.

[124] Wei Chen and Faez Ahmed. PaDGAN: Learning to Generate High-Quality Novel Designs. ASME Journal of Mechanical Design, 143(3):031703, 2021.

[125] Wei Chen and Mark Fuge. Synthesizing Designs With Interpart Dependencies Using Hierarchical Generative Adversarial Networks. ASME Journal of Mechanical Design, 141(11):111403, 2019.

[126] Sangeun Oh, Yongsu Jung, Seongsin Kim, Ikjin Lee, and Namwoo Kang. Deep Generative Design: Integration of Topology Optimization and Generative Models. ASME Journal of Mechanical Design, 141(11):111405, 2019.

[127] Amin Heyrani Nobari, Muhammad Fathy Rashad, and Faez Ahmed. Creativegan: editing generative adversarial networks for creative design synthesis. In Proceedings of the 2021 ASME International Design Engineering Technical Conferences and Computers and Information in Engineering Conference (IDETC/CIE), Virtual, Online, Aug. 17-20, 2021.

[128] Zijiang Yang, Xiaolin Li, L Catherine Brinson, Alok N Choudhary, Wei Chen, and Ankit Agrawal. Microstructural materials design via deep adversarial learning methodology. ASME Journal of Mechanical Design, 140(11): 111416, 2018.

[129] Dule Shu, James Cunningham, Gary Stump, Simon W Miller, Michael A Yukish, Timothy W Simpson, and Conrad S Tucker. 3D Design Using Generative Adversarial Networks and Physics-Based Validation. ASME Journal of Mechanical Design, 142(7):071701, 2020.

[130] Yan Gan, Yingrui Ji, Shuo Jiang, Xinxiong Liu, Zhipeng Feng, Yao Li, and Yuan Liu. Integrating aesthetic and emotional preferences in social robot design: An affective design approach with Kansei Engineering and Deep Convolutional Generative Adversarial Network. International Journal of Industrial Ergonomics, 83:103128, 2021.

[131] Danilo Jimenez Rezende, Shakir Mohamed, and Daan Wierstra. Stochastic backpropagation and approximate inference in deep generative models. In Proceedings of the 31st International Conference on Machine Learning (ICML), pages 1278-1286, Beijing, China, June 21-26, 2014.

[132] Filippo Chiarello, Paola Belingheri, and Gualtiero Fantoni. Data science for engineering design : State of the art and future directions. Computers in Industry, 129:103447, 2021.

[133] Seda Yilmaz, Colleen Seifert, Shanna R Daly, and Richard Gonzalez. Design Heuristics in innovative products. ASME Journal of Mechanical Design, 138(7):071102, 2016.

[134] Xiaoneng Jin, Mark Evans, Hua Dong, and Anqi Yao. Design heuristics for artificial intelligence: inspirational design stimuli for supporting UX designers in generating AI-powered ideas. In Proceedings of the 2021 CHI Conference on Human Factors in Computing Systems, pages 1-8, Yokohama, Japan, May 8-13, 2021.

[135] Katherine K Fu, Maria C Yang, and Kristin L Wood. Design Principles: Literature Review, Analysis, and Future Directions. ASME Journal of Mechanical Design, 138(10):101103, 2016.

[136] P John Clarkson, Caroline Simons, and Claudia Eckert. Predicting change propagation in complex design. ASME Journal of Mechanical Design, 126(5):788-797, 2004.

[137] Steven D Eppinger and Tyson R Browning. Design structure matrix methods and applications. MIT press, 2012.

[138] Timothy W Simpson, J Jiao, Zahed Siddique, and Katja Hölttä-Otto. Advances in product family and product platform design. Springer, 2014.

[139] Binyang Song, Jianxi Luo, and Kristin Wood. Data-driven platform design: Patent data and function network analysis. ASME Journal of Mechanical Design, 141(2):021101, 2019.

[140] M David Merrill. First principles of instruction. Educational technology research and development, 50(3):43-59, 2002.

[141] Jonathan Cagan and Alice M Agogino. Dimensional Variable Expansion-a formal approach to innovative design. Research in Engineering Design, 3(2):75-85, 1991.

[142] Udo Kannengiesser and John S Gero. Ekphrasis as a Basis for a Framework for Creative Design Processes. In International Conference on Design Computing and Cognition (DCC'18), pages 265-283, Politecnico di Milano, Italy, July 2-4, 2018. Springer. 
[143] Armand Hatchuel, Pascal Le Masson, Yoram Reich, and Eswaran Subrahmanian. Design theory: a foundation of a new paradigm for design science and engineering. Research in Engineering Design, 29(1):5-21, 2018.

[144] Yukari Nagai, Toshiharu Taura, and Futoshi Mukai. Concept blending and dissimilarity: factors for creative concept generation process. Design Studies, 30(6):648-675, 2009.

[145] Yuejun He. Combinational Creativity: Theories, Methods and Tools. PhD thesis, Singapore University of Technology and Design, 2019. 